\title{
Superalkali-Alkalide Interactions and Ion Pairing in Low-Polarity Solvents
}

René Riedel,* Andrew G. Seel,* Daniel Malko, Daniel P. Miller, Brendan T. Sperling, Heungjae Choi, Thomas F. Headen, Eva Zurek, Adrian Porch, Anthony Kucernak, Nicholas C. Pyper, Peter P. Edwards, * and Anthony G. M. Barrett*

Cite This: J. Am. Chem. Soc. 2021, 143, 3934-3943

Read Online

ACCESS | 네 Metrics \& More | 回 Article Recommendations | sl Supporting Information

ABSTRACT: The nature of anionic alkali metals in solution is traditionally thought to be "gaslike" and unperturbed. In contrast to this noninteracting picture, we present experimental and computational data herein that support ion pairing in alkalide solutions. Concentration dependent ionic conductivity, dielectric spectroscopy, and neutron scattering results are consistent with the presence of superalkali-alkalide ion pairs in solution, whose stability and properties have been further investigated by DFT calculations. Our temperature dependent alkali metal NMR measurements reveal that the dynamics of the alkalide species is both reversible and thermally activated suggesting a complicated exchange process for the ion paired species. The results of this study go beyond a picture of alkalides being a "gaslike" anion in solution and highlight the significance of the interaction of the alkalide with its complex countercation (superalkali).

\section{INTRODUCTION}

Anionic forms of the electropositive Group I metals, with the exception of lithium, can be generated in condensed phases. ${ }^{1}$ Termed alkalides, these monoanions are chemically highly reducing and possess a diffuse, closed-shell $n \mathrm{~s}^{2}$ configuration, resulting in an exceptionally high electronic polarizability. The formation and stabilization of alkalide species requires stringent chemical environments and involves either a disproportionation or the reduction of one elemental alkali metal by another. ${ }^{2}$ The dissolution and reduction process is facilitated by strong stabilization of the alkali cation by preorganized complexants such as crown ethers and cryptands. ${ }^{3}$ This enables alkali metals to be dissolved in even weakly polar solvents such as tetrahydrofuran (THF) by formation of alkalide anions that persist in the absence of any better electron receptor, as with ammonia or small amines (metal-ammonia solutions $^{4-6}$ and solutions containing solvated electrons ${ }^{7,8}$ ), functional groups in organic or organometallic molecules (the dissolving metal reduction), or simply a different, more reducible metal. Cryogenic temperatures are also necessitated to avoid reduction and decomposition of solvent.

Perhaps the most puzzling aspect of alkalides, which has persisted almost from the time of their discovery, is an understanding of the precise nature of their diffuse $n s^{2}$ orbital in solution. The NMR signatures of an alkalide species in solution, and in the crystalline solids they form, are significantly shielded and exhibit an exceptionally narrow line

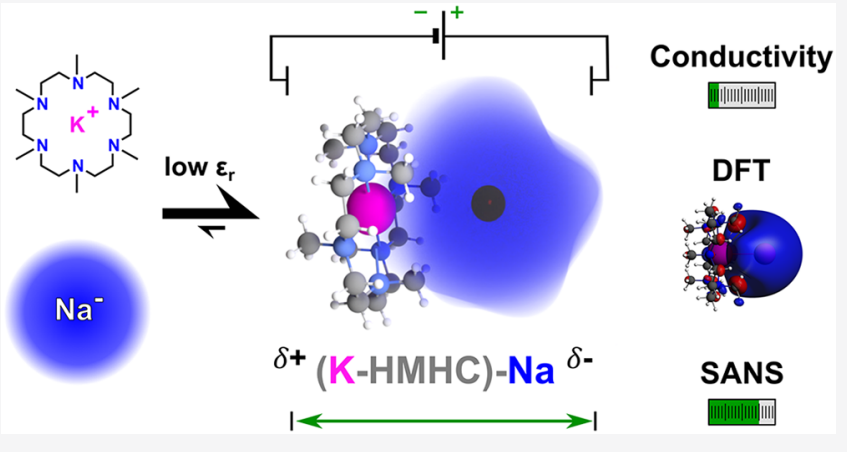

width. Considering that the alkali metals all possess quadrupolar nuclei, these features have been ascribed to the high shielding and high symmetry of an unperturbed "gaslike" anion in solution, with little to no interaction with its local environment. $^{9-14}$ However, the high polarizability of the alkalides and ready electron dissociation into solvated-electron species with increasing solvent polarity imply that the genuine alkalide could be significantly perturbed in certain cases. Indeed, the "gaslike" picture of alkalides in solution has recently been questioned by $a b$ initio calculations, which have instead provided two insights in favor of a picture of an alkalide interacting with its environment: First, it was suggested that the most stable species were formed via the association of the alkalide anion with solvated/complexed alkali cations in a known alkalide-forming solvent, 1,2-ethylenediamine, ${ }^{15}$ and it was shown that the simulated absorption spectra for such interacting species in the visible and ultraviolet ranges were in agreement with experimental observations. The complexed alkali metal cations have been termed "superalkali" cations because their LUMOs are highly expanded but retain similar

Received: January 5, 2021

Published: March 4, 2021 
symmetries to those of the uncomplexed alkali metal cations and they are able to accept electron density from the alkalide in a weak donor-acceptor sense. Second, molecular dynamics simulations on explicitly solvated sodide $\left(\mathrm{Na}^{-}\right)$ions suggested that the expanded $3 s^{2}$ orbital is perturbed by its environment, but the NMR response for the sodium nucleus is negligibly affected, despite its quadrupolar nature. ${ }^{16}$

Here, we provide experimental evidence that alkalides interact with their environment through the formation of ion paired species in solution (see Figure 1). Further support is

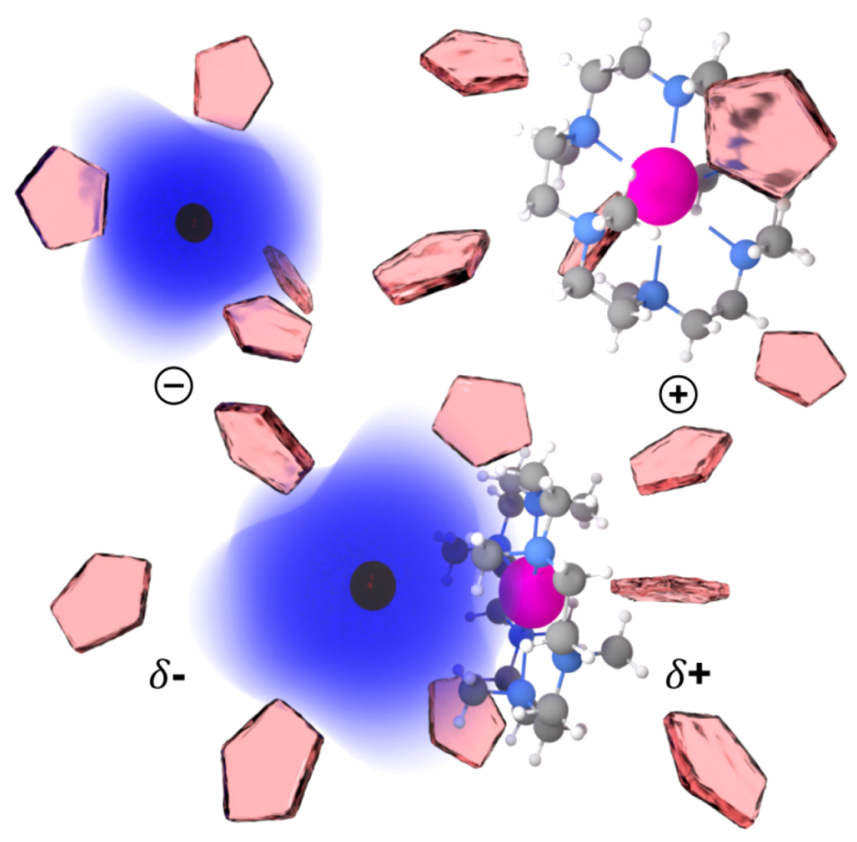

Figure 1. Illustration of potential components of a sodide solution in HMHC/THF. Separately solvated complexed potassium (pink) cation (top right) and alkalide anion (top left) with its diffuse $3 \mathrm{~s}^{2}$ valence orbital (blue) and a contact ion pair (bottom) in a medium of THF molecules (red) are indicated.

provided by density functional theory (DFT) calculations that suggest a nature beyond that of classic ion associates. ${ }^{17}$ Such weakly covalent interactions between the alkalide and the counter superalkali cation reflect a subtle chemistry for alkalides, which has previously not been reported. As such, our findings have implications for future control of alkalide properties and their potential use in photo- and electrochemical applications.

\section{EXPERIMENTAL SECTION}

Synthesis. Full experimental details and characterization regarding the preparation of $\mathrm{HMHC}$ are provided in the Supporting Information. Crude HMHC and commercially available 15-crown-5 were purified by Kugelrohr distillation from $\mathrm{NaK}$, kept under an argon atmosphere and handled inside an argon glovebox.

Ionic Conductivity Measurements. Impedance measurements were carried out using a potentiostat Gamry Reference 600 and using platinized platinum electrodes (cell constant $0.98 \mathrm{~cm}$ ). The Walden product was calculated using the shear viscosity of metal-free solutions of 15-crown-5 in THF at all relevant concentrations as an approximation of the viscosity of metal solutions. The shear viscosity was determined over the temperature range of $15-40{ }^{\circ} \mathrm{C}$ using a RheoSense $\mathrm{m}$-VROC viscometer. In a typical experiment, $\mathrm{NaK}$ was added in small portions $(<20 \mathrm{mg})$ to a solution of $0.06 \mathrm{M} 15$-crown-5 or $0.03 \mathrm{M} \mathrm{HMHC}$ in dry THF at $243 \mathrm{~K}$ while the conductivity of the mixture was monitored in set intervals of $0.5 \mathrm{~min}$. Macrocycle concentration was doubled when the concentration of dissolved metal reached approximately $70 \%$ saturation to maintain a sufficient metal dissolution rate. The ratio between metal and macrocycle concentration at a given metal concentration was found to have no effect on the conductivity.

Small Angle Neutron Scattering (SANS). SANS spectra were collected at the neutron diffractometer NIMROD ${ }^{18}$ at the ISIS Neutron and Muon Source Facility, U.K. Samples were prepared by mixing of all the components (HMHC (1 equiv) or 15-crown-5 (2 equiv), $d_{8}$-THF, $\mathrm{NaK}(n / n 1: 1,3$ equiv) $)$ in a quartz NMR tube inside an argon glovebox with a cryogenic and inert atmosphere being maintained. Samples were then warmed to $240 \mathrm{~K}$, and equilibrated for $10 \mathrm{~h}$ prior to measurement to ensure complete metal dissolution up to a point of saturation. Data were reduced using standardized procedures within the GudrunN software. ${ }^{19}$ Density values were precisely determined for all relevant metal-free complexant solutions in protiated THF using a 4 place digital LiquiPhysics Excellence density meter DM40 over the temperature range of $273-303 \mathrm{~K}$. Density values at $243 \mathrm{~K}$ were determined by linear extrapolation (Details are provided in the Supporting Information.).

Density Functional Theory. The DFT calculations used the Perdew-Burke-Ernzerhof (PBE) functional ${ }^{20}$ with the Amsterdam Density Functional (ADF) $)^{21-23}$ software package. TZP basis sets from the ADF basis set library were used for the $\mathrm{Na}, \mathrm{K}, \mathrm{C}, \mathrm{N}$, and $\mathrm{O}$ atoms, while the QZ3P + 1 diffuse function basis set was used for the $\mathrm{H}$ atoms. ${ }^{24}$ The core electronic states were kept frozen for all atoms except $\mathrm{Na}$ and $\mathrm{K}$. Further computational details are provided in the Supporting Information, section S7.

NMR Spectroscopy. ${ }^{23} \mathrm{Na}$ NMR spectra were acquired at 106 $\mathrm{MHz}$ on a Bruker DRX-400 spectrometer. ${ }^{39} \mathrm{~K}$ NMR spectra were acquired at $28 \mathrm{MHz}$ on a Bruker Avance $600 \mathrm{MHz}$ NMR spectrometer. Chemical shifts are reported as $\delta$-values in ppm relative to the cation signal from external aqueous solutions of the respective chloride salt at room temperature. Samples were prepared by addition of all components (complexant HMHC (1 equiv) or 15-crown-5 (2 equiv), $d_{8}$-THF, NaK (3 equiv)) to an oven- and flame-dried borosilicate NMR tube with a J. Young valve inside an argon glovebox. The sealed tubes were removed from the glovebox and cooled to $195 \mathrm{~K}$ before exposure of the metal alloy to the solution. The samples were stored at $195 \mathrm{~K}$ and warmed to $240 \mathrm{~K}$ for $10 \mathrm{~h}$ in preparation for the respective NMR experiment to ensure complete metal dissolution up to a point of saturation. The probe of the NMR spectrometer was cooled to $200 \mathrm{~K}$ prior to quick sample loading. The steady reduction in signal intensity upon thermal cycling is reversible, while taking into account a slight loss of intensity over time due to minor decomposition processes. All measurements were corrected for any loss in signal intensity due to a shift of the Boltzmann distribution of spin states.

\section{RESULTS AND DISCUSSION}

Design and Control of Chemical Composition. The accurate preparation and investigation of alkalide solutions, especially at concentrations as low as those shown in Figure 2a, requires the use of complexing agents that are resistant to irreversible reductive ring scission. A milestone in the development of more stable alkalide systems was the introduction of per-alkylated polyamine ligands to the field. ${ }^{25}$ This showed that the hexa-aza-crown 1,4,7,10,13,16-hexamethyl-1,4,7,10,13,16-hexaazacyclooctadecane (hexamethyl-hexacyclen, HMHC, see Figure 2b) significantly outperforms the reportedly most stable crown ether 15 -crown- $5^{26}$ in its resistance to reductive decomposition in the presence of alkalides. THF was found to be the most suitable organic solvent as it allowed for a comparably rapid metal dissolution and for the preparation of alkalide solutions with exceptionally high metal concentrations, long-lived stability, and persistence. Figure $2 \mathrm{~b}$ highlights the stark contrast in stability between the 
a Conductivity $[\mu \mathrm{S} / \mathrm{cm}]$ vs.

Concentration $[\mathrm{ppm}]$
$2.0 \mid 0.5$

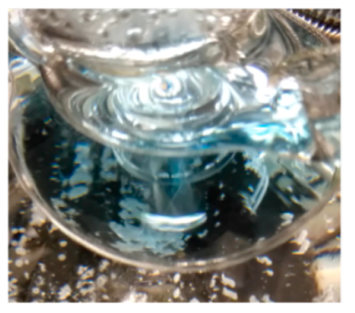

$3.2 \mid 0.9$

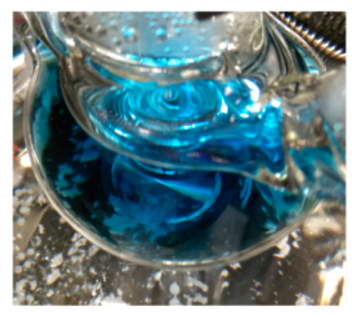

$4.0 \mid 1.2$

$5.5 \mid 1.9$

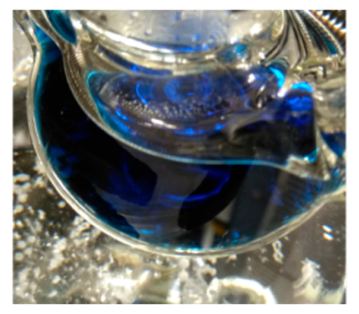

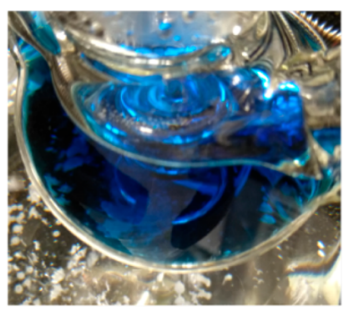

b Stability at RT $15 \mathrm{C} 5$ vs. HMHC
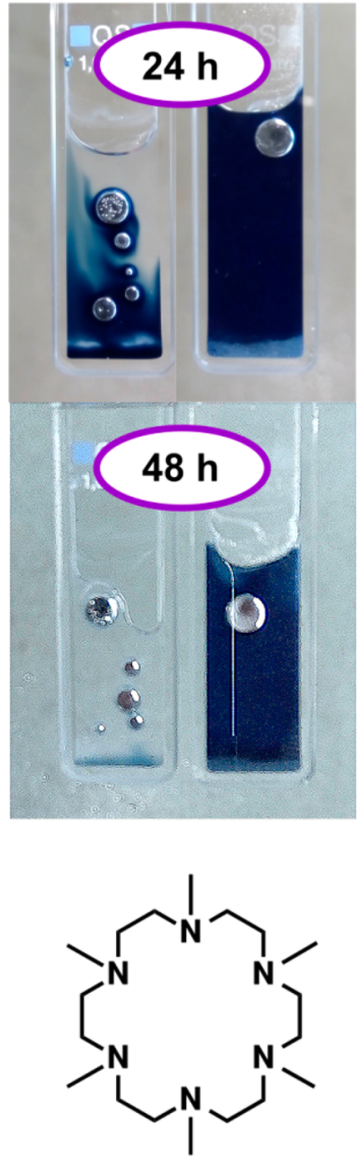

HMHC

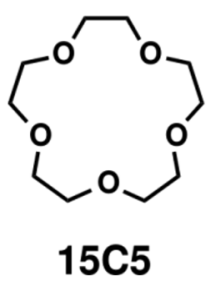

Figure 2. Alkalide solutions in low-polarity solvent tetrahydrofuran (THF). (a) Relation between concentration, conductivity, and color intensity of characteristically blue sodide solutions in $50 \mathrm{~mL}$ of THF at extremely high dilution. (b) Stability of solutions of $\mathrm{NaK}$ in $40 \mathrm{mM}$ 15-crown-5/THF (left) and $20 \mathrm{mM} \mathrm{HMHC/THF} \mathrm{(right)} \mathrm{in} \mathrm{the}$ presence of $\mathrm{NaK}$ after storage at room temperature for $24 \mathrm{~h}$ (top) and $48 \mathrm{~h}$ (bottom). Structures of aza-crown HMHC and crown ether 15crown-5 (15C5) used in this work.

metal solutions using HMHC and the more labile complexant 15-crown-5 in THF, which was ascribed to the decreased reactivity of $\mathrm{C}-\mathrm{N}$ bonds as compared to $\mathrm{C}-\mathrm{O}$ bonds under strongly reducing conditions.

Ionic Conductivity and Small Angle Neutron Scattering (SANS). Investigation of the concentration dependence of ionic conductivity of electrolyte solutions is the acknowledged technique to interrogate the nature of ion association in solution. Our experimental methodology allowed for the reproducible preparation of highly dilute metal solutions
(Figure 2a) and the accurate determination of their metal content by ion chromatography, enabling the investigation of conductivity across a wide concentration range. Plots of the relationship between concentration and the associated Walden product for solutions of $\mathrm{NaK}$ in 15-crown-5/THF and HMHC/THF (Figure 3) show that both exhibit a rapid decrease followed by a slight increase of the derived Walden product with increasing metal concentration. While the decrease in molar conductivity is a familiar consequence of ion association, the presence of a minimum at $\sim 15 \mathrm{mM}$ distinguishes the Walden product trend of the alkalide systems from that of a classic weak electrolyte. Such conductance minima have been reported for several solutions of electrolytes and ionic liquids in low-polarity solvents ${ }^{27-37}$ and agree with the theory of a feedback between the ion association equilibrium and the overall relative permittivity of mixtures in low-polarity solvents. ${ }^{31,38-42}$ Examination of the frequencydependent dielectric spectra and the static permittivity over the period of metal dissolution yields results that are consistent with an increase of the overall relative permittivity of the solution due to an increasing number of ion pairs upon metal dissolution (Supporting Information, Figure SI-4).

We employed neutron scattering measurements to examine any structural signatures for such associations. The length scales of the macrocycle-encapsulated cation or the envisaged contact-ion/ion paired superalkali-alkalide correspond to a range in reciprocal space that is intermediary between that probed in small and wide-angle neutron scattering, with intramolecular distances overlapping with intermolecular, solvent-solvent, or solvent-solute distances. The use of a protiated macrocycle and deuterated solvent overcomes this problem due to the large difference in coherent neutron scattering length of the proton $(-3.74 \mathrm{fm})$ and the deuteron $(6.67 \mathrm{fm})$. This enables the macrocyclic complex to behave as a scattering object in the limit of low scattering angle $Q$ and any change to its size/solvation via the association of the alkalide should be apparent. Within the low- $Q$ limit, the coherent differential neutron scattering cross section, $\mathrm{d} \sigma / \mathrm{d} \Omega$, is given by

$$
\frac{\mathrm{d} \sigma}{\mathrm{d} \Omega}=N V_{\mathrm{P}}^{2}(\Delta \rho)^{2} P(Q)
$$

where $N$ is the number of scattering objects of volume $V_{\mathrm{P}}, \Delta \rho$ is the contrast between the scattering-length density of the object and the average scattering-length density of the solvent, and $P(Q)$ is the form- or shape-factor for the object. This expression holds only for the dilute limit, where there are no significant "object-object" correlations in the solution.

Figure 4 presents neutron scattering data for both alkalide solutions (15-crown-5 or HMHC in THF with and without dissolved $\mathrm{K}^{+} \mathrm{Na}^{-}$) and control solutions of either 15-crown-5 or $\mathrm{HMHC}$ in $\mathrm{D}_{2} \mathrm{O}$ both with and without dissolved $\mathrm{K}^{+} \mathrm{I}^{-}$. These control solutions were chosen to represent fully iondissociated systems of separately solvated macrocycle- $\mathrm{K}^{+}$and $\mathrm{I}^{-}$. Furthermore, the iodide and sodide anions are almost identical in size and possess similar coherent neutron scattering lengths.

It is apparent from Figure 4 that the alkalide solutions both possess an increase in small-angle intensity whereas the control systems containing iodide do not exhibit such an increase. Importantly, this indicates that the scattering behavior of the macrocycle complex does not change in the control (as expected from simply binding $\mathrm{K}^{+}$with its extremely low 
a

Concentration $\left(\mathrm{mmol} \mathrm{L}^{-1}\right)$

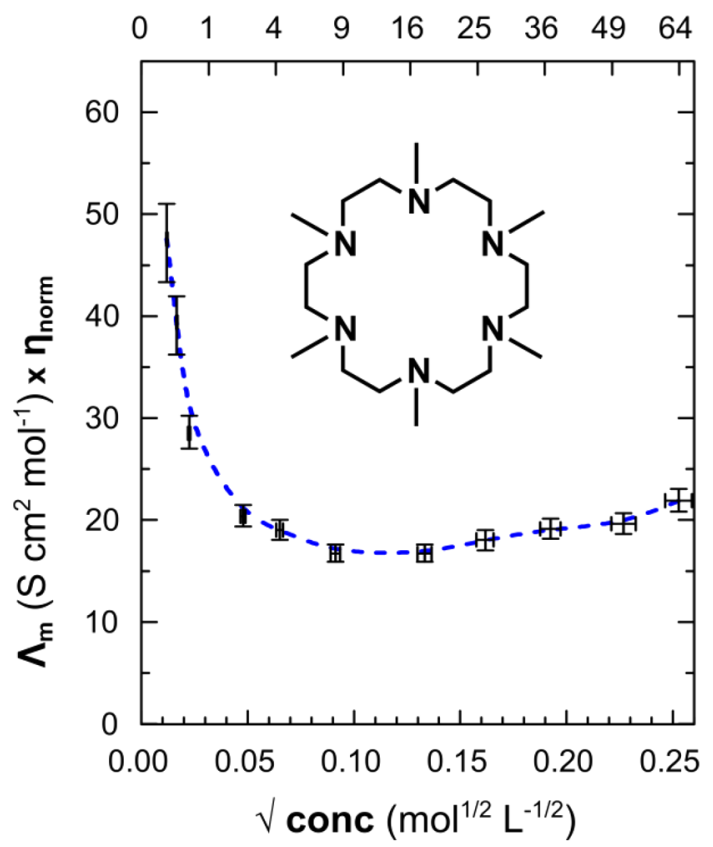

b

Concentration ( $\mathrm{mmol} \mathrm{L}^{-1}$ )

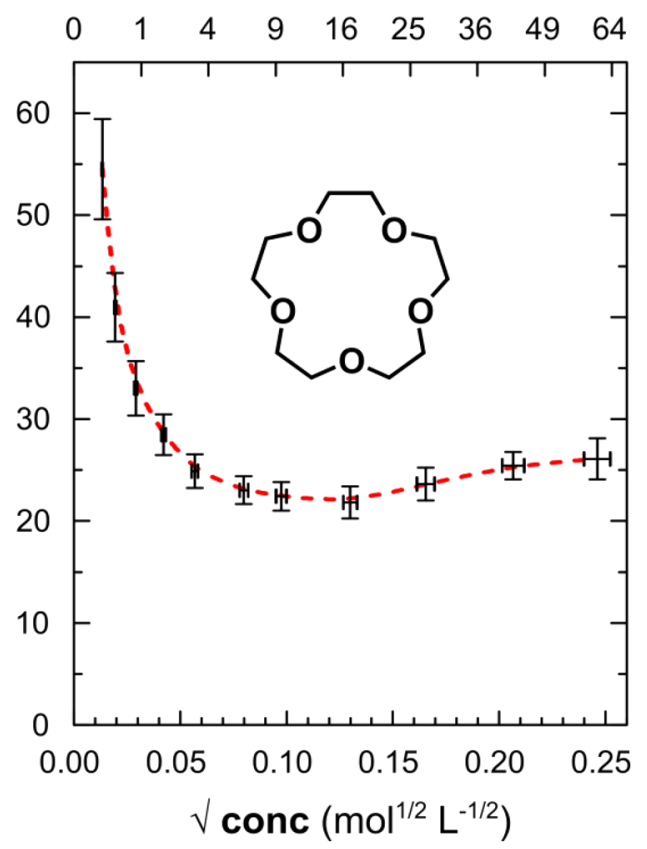

Figure 3. Walden product vs square-root of the concentration of sodide solutions. (a) Concentration dependent Walden product of solutions of $\mathrm{NaK}$ in HMHC in THF at $243 \mathrm{~K}$. (b) Concentration dependent Walden product of solutions of NaK in 15 -crown-5 in THF at $243 \mathrm{~K}$. Dashed lines represent guides to the eye.
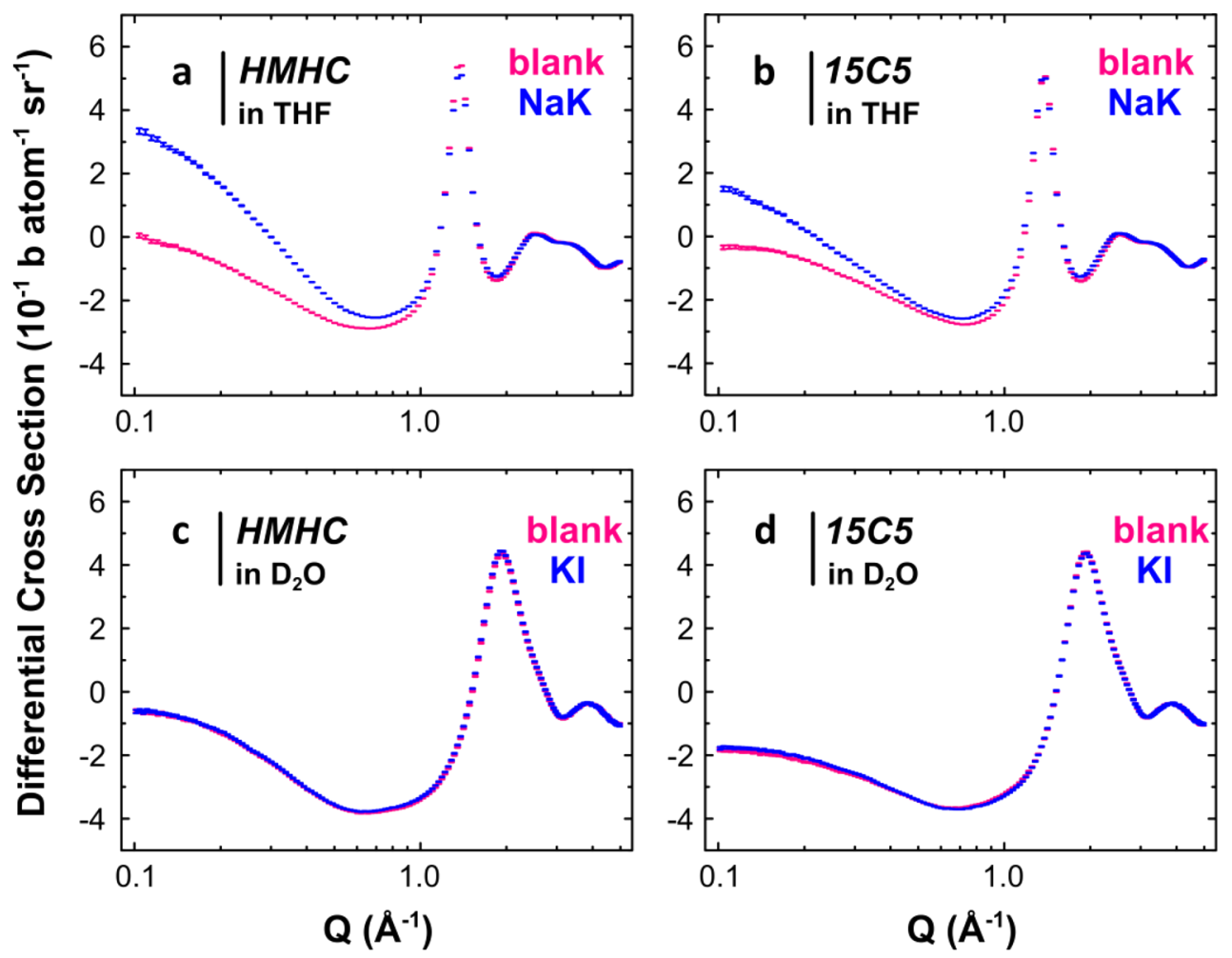

Figure 4. Small angle neutron scattering spectra for macrocycle solutions of both $\mathrm{NaK}$ in THF and $\mathrm{KI}$ in $\mathrm{D}_{2} \mathrm{O}$. Coherent differential cross section of blank (pink) and ion-containing (blue) solutions involving HMHC in THF- $d_{8}(0.05 \mathrm{M}, \mathrm{a}), \mathrm{HMHC}$ in $\mathrm{D}_{2} \mathrm{O}(0.05 \mathrm{M}, \mathrm{c}), 15-\mathrm{crown}-5$ in THF- $d_{8}$ $(0.1 \mathrm{M}, \mathrm{b})$, and 15-crown-5 in $\mathrm{D}_{2} \mathrm{O}(0.1 \mathrm{M}, \mathrm{d})$.

neutron scattering length and negligible contribution to scattering from the macrocycle complex), whereas in the alkalide systems the scattering volume and contrast has clearly increased. Simple ellipsoidal form factor models are reported in the Supporting Information in Figure SI-5 and are concordant with an association of the alkalide with the macrocyclic superalkali, effectively increasing the effective volume of the scattering object in solution from that of a solvated macrocycle 
Table 1. Binding Energies and Select Molecular Properties of Superalkali and Superalkali-Alkalide Pair Models from DFT Calculations $^{a}$

\begin{tabular}{|c|c|c|c|c|c|}
\hline & $\begin{array}{l}\text { binding energy }^{b} \\
(\mathrm{~kJ} / \mathrm{mol})\end{array}$ & $\begin{array}{c}\mathrm{M}-\mathrm{M} \text { distance } \\
(\AA)\end{array}$ & $\begin{array}{l}\text { superalkali Hirshfeld charge } \\
(\mathrm{e})\end{array}$ & $\begin{array}{l}\text { alkalide Hirshfeld charge } \\
\text { (e) }\end{array}$ & $\begin{array}{l}\text { dipole moment } \\
\text { (D) }\end{array}$ \\
\hline \multicolumn{6}{|c|}{ Superalkali M-15-crown- $5_{2}$ Models } \\
\hline $\mathrm{Na}-15$-crown- $5_{2}$ & -84.5 & & +0.26 & & 4.5 \\
\hline K-15-crown- $5_{2}$ & -109.7 & & +0.23 & & 2.7 \\
\hline \multicolumn{6}{|c|}{ Superalkali-Alkalide Pair M-15-crown-5 $(M)$ Models } \\
\hline $\mathrm{Na}-15-$ crown- $5_{2}(\mathrm{Na})$ & -147.4 & 5.71 & +0.28 & -0.45 & 17.1 \\
\hline $\begin{array}{l}\text { K-15-crown- } 5_{2}(\mathrm{Na})- \\
\text { equatorial }\end{array}$ & -178.2 & 5.59 & +0.28 & -0.42 & 15.6 \\
\hline K-15-crown $-5_{2}(\mathrm{Na})$-axial & -177.7 & 6.20 & +0.27 & -0.43 & 18.3 \\
\hline Na-15-crown- $5_{2}(\mathrm{~K})$ & -132.9 & 6.38 & +0.28 & -0.42 & 17.0 \\
\hline K-15-crown-5 $2(\mathrm{~K})$ & -166.7 & 7.08 & +0.28 & -0.43 & 17.9 \\
\hline \multicolumn{6}{|c|}{ Superalkali M-HMHC Models } \\
\hline $\mathrm{Na}-\mathrm{HMHC}$ & -62.7 & & +0.31 & & 0 \\
\hline K-HMHC & -90.9 & & +0.26 & & 0 \\
\hline \multicolumn{6}{|c|}{ Superalkali-Alkalide Pair M-HMHC (M) Models } \\
\hline $\mathrm{Na}-\mathrm{HMHC}(\mathrm{Na})$ & -152.0 & 3.61 & +0.22 & -0.23 & 9.3 \\
\hline K-HMHC (Na)-chair ${ }^{c, d}$ & -177.0 & 4.11 & +0.24 & -0.29 & 10.6 \\
\hline K-HMHC (Na)-boat ${ }^{c, d}$ & $-183.5(-150.6)$ & $4.00(4.50)$ & $+0.21(+0.27)$ & $-0.29(-0.33)$ & $10.5(12.6)$ \\
\hline Na-HMHC (K) & -130.8 & 4.36 & +0.25 & -0.21 & 9.8 \\
\hline K-HMHC (K) & -159.0 & 4.73 & +0.25 & -0.24 & 9.9 \\
\hline
\end{tabular}

${ }^{a} \mathrm{M}$ is the symbol for the alkali metal, to represent either $\mathrm{Na}$ or $\mathrm{K}$. Each HMHC system given was modeled with the chair conformation, except for the "K-HMHC ( $\mathrm{Na}$ )-boat" where the most stable and least stable positions of the sodide are given outside and within the parentheses, respectively. Each 15 -crown- $5_{2}$ system was modeled as the equatorial pair unless labeled differently. M-HMHC and $\mathrm{M}-15$-crown- $5_{2}$ are referred to as superalkalis. ${ }^{b_{\text {The }}}$ The binding energies (BE in $\mathrm{kJ} / \mathrm{mol}$ ), calculated in the gas-phase by subtracting the energy of the alkali metal(s) and 15 -crown-5/ HMHC from the total system energy (see the Supporting Information, eqs S1, S2, S4, and S5). ${ }^{c}$ The BEs were calculated using the HMHC conformation adopted in the M-HMHC $(\mathrm{M})$ model. ${ }^{d} E[\mathrm{~K}-\mathrm{HMHC}(\mathrm{Na})-$ chair $]-E[\mathrm{~K}-\mathrm{HMHC}(\mathrm{Na})-\mathrm{boat}]=0.84 \mathrm{~kJ} / \mathrm{mol}$.

to a solvated superalkali-alkalide ion pair. Moreover, a larger aggregation of ions in solution would lead to a small angle signal orders of magnitude higher than those witnessed in the alkalide solutions, thus enabling us to rule out any macroscopic phase separation or higher alkalide agglomerates in the solutions.

$\boldsymbol{A} \boldsymbol{b}$ Initio Calculations. To assess the possible species that may form in alkalide solutions, DFT calculations were performed on sodium and potassium superalkalis of HMHC and 15-crown-5, superalkali-alkalide ion pairs, and superalkali dimers. As shown in Table $1, \mathrm{~K}$ was calculated to interact more strongly than $\mathrm{Na}$ with both HMHC and 15-crown-5. The computed binding energies (BEs) suggest that the metal atoms prefer to be coordinated to two 15-crown-5 molecules as opposed to a single complexant (Supporting Information, section S15). In agreement with prior DFT calculations, ${ }^{15}$ the superalkali dimers were found to be less stable than the superalkali-alkalide ion pairs when solvation effects were considered (Supporting Information, section S14). Therefore, in this discussion we focus on the superalkali-alkalide ion pairs whose computed BEs and selected properties are given in Table 1, with optimized geometries and molecular orbital isosurfaces illustrated in Figures 5 and 6. Out of the four metal combinations that are possible, the encapsulated potassium and anionic sodide models, K-HMHC ( Na), and K-15-crown$5_{2}(\mathrm{Na})$, each possess the largest BEs, in agreement with the experimental observation of a predominance of potassium cations and sodide anions.

The flexibility of the macrocycles and approach of the second metal ion allows for several conformationally and topologically different superalkali-alkalide contact ion pairs. In its complexes with alkali cations, HMHC can adopt a conformation with all six nitrogen atoms in one plane, similar to the chair conformation in the crystalline salt of K-HMHC with tetraphenylborate ${ }^{43}$, or a boat conformation as is the case for the crystalline sodide K-HMHC $(\mathrm{Na})^{44,45}$ (Figure 5). For the 15-crown-5 systems, an alkalide can approach either axial or equatorial to the superalkali complex (Figure 6). These two systems are essentially isoenergetic, although the equatorial approach is statistically preferred. The chair and boat forms of K-HMHC $(\mathrm{Na})$ are also isoenergetic, but pure HMHC favors the chair conformation by $5.9 \mathrm{~kJ} / \mathrm{mol}$. Calculations exploring the potential energy landscape associated with flipping the HMHC between the chair and boat conformations, both for pure HMHC and within the superalkali-alkalide complex, concluded that the chair form is the dominant species in solution (Supporting Information, section S16). Therefore, we focus upon the chair HMHC in this discussion.

Turning to the NMR parameters, the computed shielding constant for the sodide in K-HMHC (Na) is 40-46 ppm larger than for the sodium cation in $\mathrm{Na}-\mathrm{HMHC}(\mathrm{K})$ or $\mathrm{Na}$ HMHC $(\mathrm{Na})$. The Hirshfeld charges capture the loss of electron density from the encapsulated metal atom and an increase in electron density on the surrounding metal for all ion paired systems, as expected for the alkalide state being maintained even in a close ion pair. Both K-HMHC $(\mathrm{Na})$ and $\mathrm{K}-15$-crown $5_{2}(\mathrm{Na})$ possess significant dipole moments, in line with the rationale for the experimentally observed increase in molar conductivity at higher alkalide concentrations (Figure 3). This further rules out weakly dipolar superalkali dimers (Supporting Information Table SI-4).

The chair HMHC superalkali $C_{i}$ symmetry SOMO extends from the metal symmetrically surrounding the superalkali above and below, in contrast to the 15 -crown- $5_{2}$ superalkali $C_{1}$ symmetry SOMO that persists about the ether rings but does not have much character near the alkali metal (cf. Figures 5 and 
a

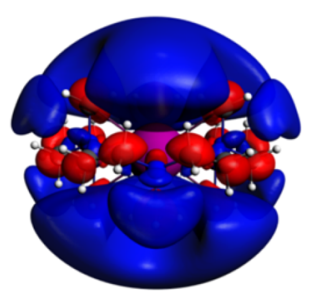

b

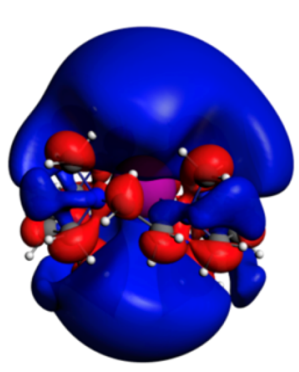

C

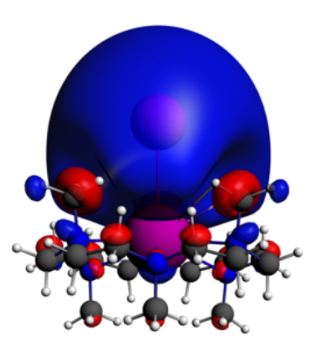

e

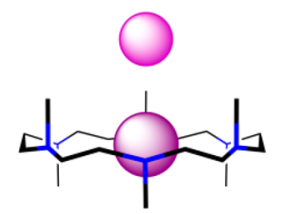

d

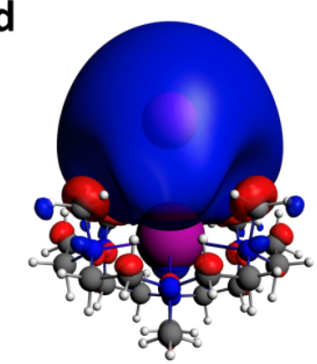

f

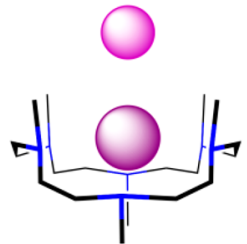

Figure 5. Computed SOMOs/HOMOs of superalkali K-HMHC and superalkali-alkalide $(\mathrm{K}-\mathrm{HMHC})^{\delta+}(\mathrm{Na})^{\delta-}$. Isosurfaces (isovalue = $\pm 0.010 \mathrm{au}$ ) for superalkali models K-HMHC in the chair (a) and boat (b) conformations and for the superalkali-alkalide models $\mathrm{K}$ HMHC $(\mathrm{Na})$ in the chair $(\mathrm{c})$ and boat $(\mathrm{d})$ conformations. Ion pairs in the chair (e) and boat (f) conformations illustrated in the form of a cartoon.

a

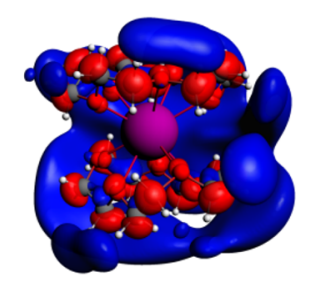

C

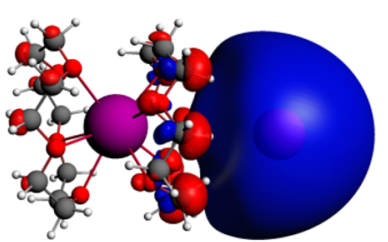

b

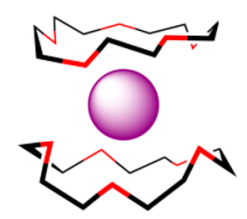

d

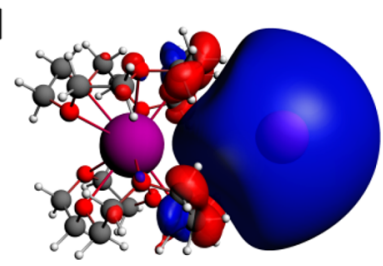

Figure 6. Computed SOMO/HOMOs of superalkali K-15-crown- $5_{2}$ and superalkali-alkalide $\left(\mathrm{K}-15-\text { crown }-5_{2}\right)^{\delta+}(\mathrm{Na})^{\delta-}$. Isosurfaces (isovalue $= \pm 0.010 \mathrm{au})$ for superalkali model K-15-crown- $5_{2}$ (a) and superalkali-alkalide $\mathrm{K}-15$-crown- $5_{2}(\mathrm{Na})$ with the alkalide in the axial (c) and equatorial (d) positions with respect to the sandwich complex (as illustrated in the form of a cartoon (b)).

6). The HOMOs of the superalkali-alkalide complexes are concentrated on the sodide component, and they have a large spatial extent past the alkalide portion of the ion pair, in

agreement with our neutron scattering results and with the crystallographically derived radii of alkalides in the solid state. These HOMOs are formed from a bonding in-phase interaction between the SOMO of the sodium atom with the SOMO of the potassium superalkali, which itself contains character from the LUMOs of the complexant, as well as $4 \mathrm{~s}$ and $5 \mathrm{~s} \mathrm{~K}$ orbitals. The bond orders of 0.65 and 0.57 computed between $\mathrm{K}$ and $\mathrm{Na}$ highlight the partial covalency of the interaction in K-HMHC (Na) and K-15-crown-5 $5_{2}(\mathrm{Na})$, respectively. Additional stability in these superalkali and superalkali-alkalide complexes, alongside coordination and $\mathrm{M}^{\delta+} \mathrm{M}^{\delta-}$ pairing, originates from intramolecular $\mathrm{H} \rightsquigarrow \rightsquigarrow \mathrm{H}$ interactions first introduced in ref 5 . The $\mathrm{H} \leftrightarrow m \rightarrow \mathrm{H}$ interaction is mediated through orbital overlap between neighboring hydrogen atoms, as seen in the superalkali SOMOs and superalkali-alkalide HOMOs (Figures 5, 6, and Supporting Information Figures SI-9-SI-14, SI-17). This $\mathrm{H} \leftrightarrow \rightsquigarrow \mathrm{H}$ bonding arises from the partial population of the LUMO of the organic species when alkali metals are introduced to the system (Supporting Information Figures SI-7 and SI-8) and is reminiscent of Rydberg bonding proposed by Simons. ${ }^{46,47}$ One consequence of this $\mathrm{H} \leftrightarrow \cdots \mathrm{H}$ interaction is that the distance between pairs of hydrogen atoms is $\sim 0.1 \AA$ shorter in the superalkali systems as compared to pure HMHC and 15crown-5.

A fragment orbital analysis was performed using the (spinrestricted) distorted K-HMHC/K-15-crown $-5_{2}$ superalkalis (as found in the optimized superalkali-alkalide species) and the (spin-restricted) $\mathrm{Na}$ atoms as the fragments. ${ }^{21}$ This revealed the composition of the molecular orbitals of the complex in terms of the occupied and unoccupied orbitals of the fragments. As shown in Table SI-12, the Na 3s SOMO is the dominant contribution to the all-important HOMO of the chair K-HMHC (Na) and equatorial K-15-crown-5 $5_{2}(\mathrm{Na})$ complexes (51.8\% and $67.9 \%$, respectively) as expected. The SOMO of the superalkali provides the second largest contribution (34.2\% and $20.0 \%$ ), but diffuse unoccupied orbitals on the superalkali are also an important component, suggesting this may be another manifestation of Rydberg bonding (Supporting Information, section S19). ${ }^{46,47}$

Variable Temperature NMR Spectroscopy. ${ }^{23} \mathrm{Na}$ and ${ }^{39} \mathrm{~K}$ NMR spectra under cryogenic conditions exhibit the expected narrow, shielded signals in concentrated solutions of macrocycle $/ \mathrm{NaK}$ and macrocycle/K in $\mathrm{THF}$, respectively (Figure 7 and Supporting Information Figure SI-1). The dynamics of the alkalide species and their interactions with their local environment or with other components in solution are evidenced through comparison of ${ }^{23} \mathrm{Na}$ spectra at different temperatures over an accessible range of more than $60 \mathrm{~K}$ (Figure 7). Increasing the temperature results in a rapid and reversible decrease in the total integrated area of signals in the chemical shift range of $(-61)-(-63) \mathrm{ppm}$ for both macrocycle systems.

Considering the lack of any additional signals in the chemical shift range of $(+100)-(-200)$ ppm, we attribute the unusual behavior of alkalide species in NMR to an exchange process between an NMR-visible and an NMRinvisible species, each involving the alkali metal nucleus of interest. The line width of the alkalide signal increases only slightly from 2.9 to $9.0 \mathrm{~Hz}$ from 235 to $290 \mathrm{~K}$ despite the drastic loss in signal intensity. This suggests a thermally activated transformation of the NMR-visible species in the slow exchange limit. ${ }^{48}$ Very minor changes in the chemical 

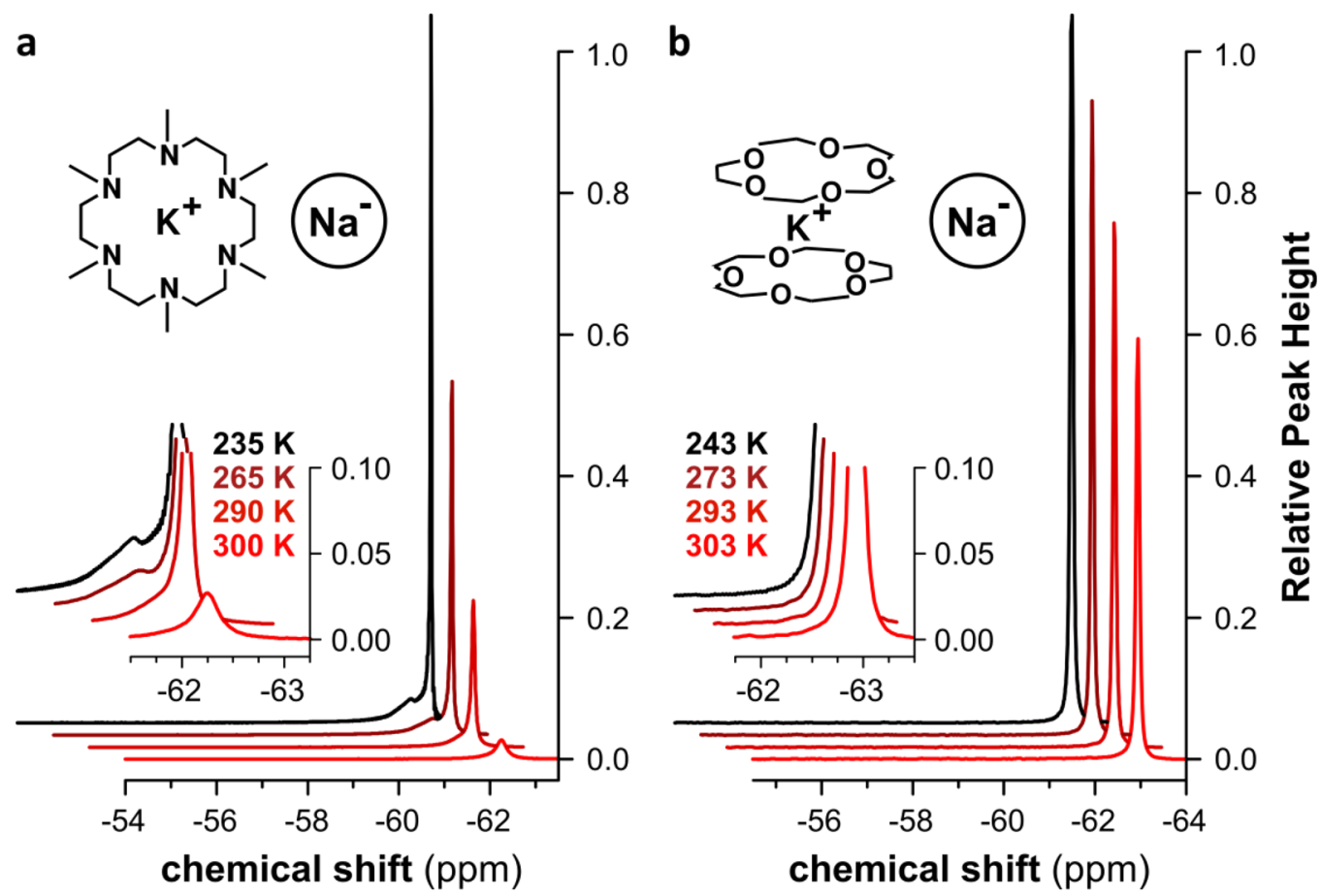

Figure 7. Temperature dependent ${ }^{23} \mathrm{Na}$ NMR spectroscopy of sodide solutions in THF. (a) Upfield range of ${ }^{23} \mathrm{Na}$ spectra showing the temperature dependent change in shape and total signal intensity of the alkalide signal of a solution of $0.3 \mathrm{M} \mathrm{NaK}$ in HMHC/THF in the chemical shift range between $(-61)-(-65) \mathrm{ppm}$. (b) Upfield range of ${ }^{23} \mathrm{Na}$ spectra showing the temperature dependent change in shape and total signal intensity of the alkalide signal of a solution of $0.4 \mathrm{M} \mathrm{NaK}$ in 15 -crown-5/THF in the chemical shift range between $(-61)-(-65)$ ppm. Spectra are referenced to $1 \mathrm{M}$ aqueous $\mathrm{NaCl}$ solution at room temperature according to IUPAC recommendations. Peak heights in both sets are normalized to their respective lowest temperatures. Insets show zooms of the signal onsets.

shift of the signals cannot be conclusively demonstrated here due to the sensitive temperature dependence of the reference signal.

If the relaxation of the quadrupolar alkalide nucleus was purely due to the approach of the superalkali countercation, it must be induced by a sufficiently large electric field gradient (EFG) at the alkalide nucleus. However, our computed EFG, $V_{z z}$, of the isolated sodide does not differ from that in the ion pair and is essentially 0 relative to the computed $V_{z z}$ for $\mathrm{Na}$ and $\mathrm{K}$ in the superalkali (Supporting Information Tables SI-5 and SI-7). Recent results from $a b$ initio molecular dynamics showed that the sodide anion may appear in NMR experiments as if it were an unperturbed, spherical ion, despite the polarizable $3 \mathrm{~s}$ orbital being strongly affected by the surrounding species in solution. ${ }^{16}$ We note that the solvent modeled in ref 16 was methylamine, a solvent of higher dielectric than the THF solutions used in this study and which would be expected to perturb the alkalide species to an even greater extent. Hence, we conclude that the NMR-visible alkalide species is subject to a fast and reversible exchange process with an NMR invisible species, which is particularly short-lived. This may be a paramagnetic species or a charge-transfer state but is not due to the association and dissociation of the superalkali-alkalide complex.

Another interesting and exclusive feature of the HMHC sodide system observed upon close inspection of the ${ }^{23} \mathrm{Na}$ NMR is the existence of a small shoulder in the major signal on the low-field side (Figure 7a). This shoulder becomes more pronounced as the signals drift apart with decreasing temperature. The small difference in chemical shift between the primary and secondary signals around the $-60 \mathrm{ppm}$ chemical shift range suggests the involvement of a coordinative or, more likely, a conformational difference between both NMR-visible species without a loss of its integrity.

Mindful of our above discussion concerning the effect of a perturbation of the alkalide on its NMR signature, we suggest that this is due to the conformational flexibility of the superalkali-alkalide complex of HMHC that is absent in the 15-crown-5 case, bearing in mind that both chairlike and boatlike conformations for the HMHC macrocycle exist in crystalline systems. Clearly, further work remains as to identifying the precise nature, and mechanisms, of these intriguing exchange processes.

\section{CONCLUSION}

Alkalides have a unique place in the history and chemistry of the s-block elements. ${ }^{1}$ Since their discovery by J. L. Dye and colleagues, the characterization of the alkalide species in condensed matter systems has led to the fascinating discussion of their diffuse, yet localized, electronic states in weakly polar solvents and indeed to the application of alkalides as highly reductive species across chemistry. ${ }^{25,49-51}$ The existence of ion paired species has been mooted since the very earliest discussions of alkalides but has never been experimentally demonstrated conclusively.

Herein, we have provided experimental evidence of the observation and effect of ion pairing of alkalides in solution, both from examination of their macroscopic conductivity and dielectric properties to the local disruption of solvent scattering density witnessed in coherent neutron scattering. To suggest what form these ion pairs may take in solution, we have carried out DFT calculations on a number of possible superalkalialkalide complexes in addition to superalkalis and superalkali dimers. In agreement with recent $a b$ initio results, ${ }^{16}$ our models 
implicate that the superalkali-alkalide complex, which we suggest is the dominant species in solution, may be indistinguishable in NMR from an isolated solvated alkalide and so we have revisited the classical interpretation of such data in the literature of alkalides. We attach great importance to the temperature dependence of the conformationally dynamic HMHC system, indicating that there is much more to be understood as to the kinetics of alkalides in solution: The reversible perturbation and possible disintegration of the NMR-visible species (that may well be the contact ion pair) highlights the significance of the interaction of alkalides with their complex countercations.

Our studies paint a picture of an alkalide species being far beyond a "gaslike" ion in solution, but instead one that could be "chemically" controlled and developed by considering superalkali-alkalide interactions of the sort delineated here. We believe that the interactions of alkalides in solution is merely the most recent in a long line of surprising and unique aspects of s-block chemistry ${ }^{1}$ and certainly one that has the potential to affect how these systems are extended and applied in the future.

\section{ASSOCIATED CONTENT}

\section{SI Supporting Information}

The Supporting Information is available free of charge at https://pubs.acs.org/doi/10.1021/jacs.1c00115.

Experimental protocols and full characterization, NMR data, description of ionic conductivity methodology, dielectric spectroscopy methodology and data, computational details and data, isosurfaces, and structure coordinates (PDF)

\section{AUTHOR INFORMATION}

\section{Corresponding Authors}

René Riedel - Department of Chemistry, Imperial College London, Molecular Sciences Research Hub, London W12 OBZ, U.K.; 이 orcid.org/0000-0003-1501-0538; Email: rene.riedel13@alumni.imperial.ac.uk

Andrew G. Seel - Department of Physics and Astronomy, University College London, London WC1E 6BT, U.K.; Inorganic Chemistry Laboratories, University of Oxford, Oxford OX1 3QR, U.K.; (1) orcid.org/0000-0003-01038388; Email: a.seel@ucl.ac.uk

Peter P. Edwards - Inorganic Chemistry Laboratories, University of Oxford, Oxford OX1 3QR, U.K.; (1) orcid.org/ 0000-0001-7340-4856; Email: peter.edwards@ chem.ox.ac.uk

Anthony G. M. Barrett - Department of Chemistry, Imperial College London, Molecular Sciences Research Hub, London W12 OBZ, U.K.; (i) orcid.org/0000-0002-8485-215X; Email: agm.barrett@imperial.ac.uk

\section{Authors}

Daniel Malko - Department of Chemistry, Imperial College London, Molecular Sciences Research Hub, London W12 OBZ, U.K.

Daniel P. Miller - Department of Chemistry, Hofstra University, Hempstead, New York 11549, United States; (1) orcid.org/0000-0003-1507-2667

Brendan T. Sperling - Department of Chemistry, Hofstra University, Hempstead, New York 11549, United States
Heungjae Choi - School of Engineering, Cardiff University, Cardiff CF24 3AA, U.K.; (1) orcid.org/0000-0003-1108293X

Thomas F. Headen - ISIS Neutron and Muon Source, Science and Technology Facilities Council, Rutherford Appleton Laboratory, Didcot OX11 OQX, U.K.; (1) orcid.org/00000003-0095-5731

Eva Zurek - Department of Chemistry, State University of New York at Buffalo, Buffalo, New York 14260-3000, United

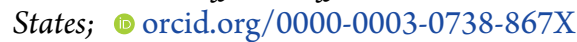

Adrian Porch - School of Engineering, Cardiff University, Cardiff CF24 3AA, U.K.

Anthony Kucernak - Department of Chemistry, Imperial College London, Molecular Sciences Research Hub, London W12 OBZ, U.K.; 1 orcid.org/0000-0002-5790-9683

Nicholas C. Pyper - University Chemical Laboratory, Cambridge CB2 1EW, U.K.

Complete contact information is available at:

https://pubs.acs.org/10.1021/jacs.1c00115

\section{Notes}

The authors declare no competing financial interest.

\section{ACKNOWLEDGMENTS}

We thank GSK for support to A.G.M.B. with the Glaxo endowment. This work is part-funded by the European Regional Development Fund through the Welsh Government. Experiments at the ISIS Neutron and Muon Source were supported by a beamtime allocation RB1810754 ${ }^{53}$ from the Science and Technology Facilities Council. D.P.M. and E.Z. thank the Center for Computational Research at the University at Buffalo, SUNY for computational support. ${ }^{52}$ D.P.M. thanks Hofstra University for funding through a Faculty Research and Development Grant (FRDG). E.Z. acknowledges the NSF (Grant DMR-1827815) for financial support and Jochen Autschbach for valuable discussions.

\section{ABBREVIATIONS}

15C5, 15-crown-5; DFT, density functional theory; EFG, electric field gradient; HMHC, 1,4,7,10,13,16-hexamethyl1,4,7,10,13,16-hexaazacyclooctadecane; HOMO, highest occupied molecular orbital; LUMO, lowest unoccupied molecular orbital; NMR, nuclear magnetic resonance; SANS, small angle neutron scattering; SOMO, singly occupied molecular orbital; THF, tetrahydrofuran

\section{REFERENCES}

(1) Dye, J. L. The alkali metals: 200 years of surprises. Philos. Trans. R. Soc., A 2015, 373, 20140174.

(2) Dye, J. L. Compounds of alkali metal anions. Angew. Chem., Int. Ed. Engl. 1979, 18, 587-598.

(3) Dye, J. L.; Redko, M. Y.; Huang, R. H.; Jackson, J. E. Role of cation complexants in the synthesis of alkalides and electrides. $A d v$. Inorg. Chem. 2006, 59, 205-231.

(4) Thompson, J. C. Electrons in Liquid Ammonia; Oxford University Press: Oxford, U.K., 1976.

(5) Zurek, E.; Edwards, P. P.; Hoffmann, R. A molecular perspective on lithium-ammonia solutions. Angew. Chem., Int. Ed. 2009, 48, $8198-8232$.

(6) Catterall, R.; Mott, N. F. Metal-ammonia solutions. Adv. Phys. 1969, 18, 665-680.

(7) Buttersack, T.; Mason, Ph. E.; McMullen, R. S.; Schewe, H. Ch; Martinek, T.; Brezina, K.; Crhan, M.; Gomez, A.; Hein, D.; Wartner, G.; Seidel, R.; Ali, H.; Thürmer, S.; Marsalek, O.; Winter, B.; 
Bradforth, S. E.; Jungwirth, P. Photoelectron spectra of alkali metalammonia microjets: From blue electrolyte to bronze metal. Science 2020, 368, 1086-1091.

(8) Hartweg, S.; West, A. H. C.; Yoder, B. L.; Signorell, R. Metal transition in sodium-ammonia nanodroplets. Angew. Chem., Int. Ed. 2016, 55, 12347-12350.

(9) Ceraso, J. M.; Dye, J. L. ${ }^{23} \mathrm{Na}$ NMR spectrum of the sodium anion. J. Chem. Phys. 1974, 61, 1585.

(10) Edwards, P. P.; Ellaboudy, A. S.; Holton, D. M. NMR spectrum of the potassium anion $\mathrm{K}^{-}$. Nature 1985, 317, 242-244.

(11) Tinkham, M. L.; Dye, J. L. First observation by potassium-39 NMR of $\mathrm{K}^{-}$in solution and in crystalline potassides. J. Am. Chem. Soc. 1985, 107, 6129-6130.

(12) Ellaboudy, A. S.; Holton, D. M.; Pyper, N. C.; Edwards, P. P.; Wood, B.; McFarlane, W. Gas like nature of the sodium anion in solution. Nature 1986, 321, 684-685.

(13) Holton, D. M.; Ellaboudy, A.; Pyper, N. C.; Edwards, P. P. Nuclear spin-lattice relaxation in the sodium anion, $\mathrm{Na}^{-}$. J. Chem. Phys. 1986, 84, 1089.

(14) Pyper, N. C.; Edwards, P. P. Nuclear shielding in the alkali metal anions. J. Am. Chem. Soc. 1986, 108, 78-81.

(15) Zurek, E. Alkali metals in ethylenediamine: A computational study of the optical absorption spectra and NMR parameters of $\left[\mathrm{M}(\mathrm{en}){ }_{3}{ }^{\delta+} \cdot \mathrm{M}^{\delta-}\right]$ ion pairs. J. Am. Chem. Soc. 2011, 133, 4829-4839.

(16) Abella, L.; Philips, A.; Autschbach, J. The sodium anion is strongly perturbed in the condensed phase even though it appears like a free ion in nuclear magnetic resonance experiments. J. Phys. Chem. Lett. 2020, 11, 843-850.

(17) Szwarc, M. Ions and ion pairs. Acc. Chem. Res. 1969, 2, 87-86.

(18) Bowron, D. T.; Soper, A. K.; Jones, K.; Ansell, S.; Birch, S.; Norris, J.; Perrott, L.; Riedel, D.; Rhodes, N. J.; Wakefield, S. R.; Botti, A.; Ricci, M.-A.; Grazzi, F.; Zoppi, M. NIMROD: The near and intermediate range order diffractometer of the ISIS second target station. Rev. Sci. Instrum. 2010, 81, 033905.

(19) Soper, A. K. GudrunN and GudrunX: Programs for Correcting Raw Neutron and X-ray Diffraction Data to Differential Scattering Cross Section, Rutherford Appleton Laboratory Technical Report No. RALTR-2011-013, 2011; https://epubs.stfc.ac.uk/.

(20) Perdew, J. P.; Burke, K.; Ernzerhof, M. Generalized gradient approximation made simple. Phys. Rev. Lett. 1996, 77, 3865-3868.

(21) Te Velde, G.; Bickelhaupt, F. M.; Baerends, E. J.; Fonseca Guerra, C.; van Gisbergen, S. J. A.; Snijders, J. G.; Ziegler, T. Chemistry with ADF. J. Comput. Chem. 2001, 22, 931-967.

(22) Fonseca Guerra, C.; Snijders, J. G.; te Velde, G.; Baerends, E. J. Towards an order-N DFT method. Theor. Chem. Acc. 1998, 99, 391403.

(23) ADF, SCM, Theoretical Chemistry; Vrije Universiteit: Amsterdam, The Netherlands, http://www.scm.com.

(24) Van Lenthe, E.; Baerends, E. J. Optimized Slater-type basis sets for the elements 1-118. J. Comput. Chem. 2003, 24, 1142-1156.

(25) Barrett, A. G. M.; Godfrey, Ch. R. A.; Hollinshead, D. M.; Prokopiou, P. A.; Barton, D. H. R.; Boar, R. B.; Joukhadar, L.; McGhie, J. F.; Misra, S. C. Dissolving metal reduction of esters to alkanes. A method for the deoxygenation of alcohols. J. Chem. Soc., Perkin Trans. 1 1981, 1501-1509.

(26) Grobelny, Z.; Stolarzewicz, A.; Sokól, M.; Grobelny, J.; Janeczek, H. Enhanced stability of potassium solutions in tetrahydrofuran containing 15-crown-5. J. Phys. Chem. 1992, 96, 5193-5196.

(27) Sakhanov, A. N. Investigations upon abnormal electrolytic dissociation. J. Phys. Chem. 1917, 21, 169-189.

(28) Sakhanov, A. N.; Prscheborovsky, J. The conductivity and dissociation of silver nitrate in solvents with dielectric constant below 13. J. Russ. Chem. Soc. 1915, 47, 849-858.

(29) Weingärtner, $\mathrm{H}$. Corresponding states for electrolyte solutions. Pure Appl. Chem. 2001, 73, 1733-1748.

(30) Petrucci, S.; Eyring, E. M. Microwave dielectric relaxation, electrical conductance and ultrasonic relaxation of $\mathrm{LiClO}_{4}$ in polyethylene oxide dimethyl ether-500 (PEO-500). Phys. Chem. Chem. Phys. 2002, 4, 6043-6046.
(31) Borodin, O.; Douglas, R.; Smith, G.; Eyring, E. M.; Petrucci, S. Microwave dielectric relaxation, electrical conductance, and ultrasonic relaxation of $\mathrm{LiPF}_{6}$ in poly(ethylene oxide) dimethyl ether-500. J. Phys. Chem. B 2002, 106, 2140-2145.

(32) Barthel, J.; Gerber, R.; Gores, H. J. The temperature dependence of the properties of electrolyte solutions VI. Triple ion formation in solvents of low permittivity exemplified by $\mathrm{LiBF}_{4}$ solutions in dimethoxyethane. Ber. Bunsenges. Phys. Chem. 1984, 88, 616-622.

(33) Chen, Z.; Hojo, M. Relationship between triple ion formation constants and the salt concentration of the minimum in the conductometric curves in low-permittivity solvents. J. Phys. Chem. B 1997, 101, 10896-10902.

(34) Bhattacharjee, A.; Roy, M. N. Ion association and solvation behavior of tetraalkylammonium iodides in binary mixtures of dichloromethane $+\mathrm{N}, \mathrm{N}$-dimethylformamide probed by a conductometric study. Phys. Chem. Chem. Phys. 2010, 12, 14534-14542.

(35) Ekka, D.; Roy, M. N. Conductance, a contrivance to explore ion association and solvation behavior of an ionic liquid (tetrabutylphosphonium tetrafluoroborate) in acetonitrile, tetrahydrofuran, 1,3dioxolane, and their binaries. J. Phys. Chem. B 2012, 116, 1168711694.

(36) Fleshman, A. M.; Petrowsky, M.; Frech, R. Application of the compensated Arrhenius formalism to self-diffusion: implications for ionic conductivity and dielectric relaxation. J. Phys. Chem. B 2013, $117,5330-5337$.

(37) Cade, E. A.; Petenuci, J., 3rd; Hoffmann, M. M. Aggregation behavior of several ionic liquids in molecular solvents of low polarityindication of a bimodal distribution. ChemPhysChem 2016, 17, 520529.

(38) Weingärtner, H.; Weiss, V. C.; Schröer, W. Ion association and electrical conductance minimum in Debye-Hückel-based theories of the hard sphere ionic fluid. J. Chem. Phys. 2000, 113, 762-770.

(39) Lee, B. S.; Lin, S. T. The origin of ion-pairing and redissociation of ionic liquid. J. Phys. Chem. B 2017, 121, 5818-5823.

(40) Cavell, E. A. S.; Knight, P. C. Effect of concentration changes on permittivity of electrolyte solutions. Z. Phys. Chem. 1968, 57, 3314.

(41) Petrucci, S.; Masiker, M. C.; Eyring, E. M. The possible presence of triple ions in electrolyte solutions of low dielectric permittivity. J. Solution Chem. 2008, 37, 1031-1035.

(42) Levin, Y.; Fisher, M. E. Criticality in the hard-sphere ionic fluid. Phys. A 1996, 225, 164-220.

(43) Dyke, J.; Levason, W.; Light, M. E.; Pugh, D.; Reid, G.; Bhakhoa, H.; Ramasami, P.; Rhyman, L. Aza-macrocyclic complexes of the group 1 cations - synthesis, structures and density functional theory study. Dalton Trans. 2015, 44, 13853-13866.

(44) Kuchenmeister, M. E.; Dye, J. L. Synthesis and structures of two thermally stable sodides with the macrocyclic complexant hexamethyl hexacyclen. J. Am. Chem. Soc. 1989, 111, 935-938.

(45) Kuchenmeister, M. E. Synthesis and characterization of new alkalides and electrides via the tertiary amine complexing agents: Steps toward thermal stability. Doctoral Thesis, Michigan State University, East Lansing, MI, 1989.

(46) Barrios, R.; Skurski, P.; Simons, J. Characterization of the Rydberg Bonding in $\left(\mathrm{NH}_{4}\right)_{2}{ }^{-}$. J. Phys. Chem. A 2000, 104, 1085510858 .

(47) Ketvirtis, A. E.; Simons, J. Dissociative Recombination of $\mathrm{H}_{3} \mathrm{O}^{+}$. J. Phys. Chem. A 1999, 103, 6552-6563.

(48) Millet, O.; Loria, J. P.; Kroenke, C. D.; Pons, M.; Palmer, A. G. The static magnetic field dependence of chemical exchange linebroadening defines the NMR chemical shift time scale. J. Am. Chem. Soc. 2000, 122, 2867-2877.

(49) Martini, I. B.; Barthel, E. R.; Schwartz, B. J. Manipulating the production and recombination of electrons during electron transfer: Femtosecond control of the charge-transfer-to-solvent (CTTS) dynamics of the sodium anion. J. Am. Chem. Soc. 2002, 124, 76227634. 
(50) Bragg, A. E.; Cavanagh, M. C.; Schwartz, B. J. Linear response breakdown in solvation dynamics induced by atomic electron-transfer reactions. Science 2008, 321, 1817-1822.

(51) MacDonald, M. R.; Bates, J. E.; Ziller, J. W.; Furche, F.; Evans, W. J. Completing the series of +2 ions for the lanthanide elements: Synthesis of molecular complexes of $\mathrm{Pr} 2+, \mathrm{Gd} 2+, \mathrm{Tb} 2+$, and $\mathrm{Lu} 2+$. J. Am. Chem. Soc. 2013, 135, 9857-9868.

(52) http://hdl.handle.net/10477/79221.

(53) Barrett, A. G. M., et al. Alkali metal anions in solution - Ion pairing and aggregation as a survival strategy. STFC ISIS Neutron and Muon Source, 2018, DOI: 10.5286/ISIS.E.RB1810754. 